\title{
Improved EMD Method for the Separation of Systematic Errors
}

\author{
Zhou Zhiheng \\ Graduate Students' Brigade \\ Naval Aeronautical and Astronautical University \\ Yantai, China \\ e-mail: zhouzhihengxf@sina.com
}

\section{Zhao Jianjun}

Department of Ordnance Science and Technology

Naval Aeronautical and Astronautical University

Yantai, China

e-mail: hyzjj@163.com

\author{
Yao Gang \\ Department of Ordnance Science and Technology \\ Naval Aeronautical and Astronautical University \\ Yantai, China \\ e-mail: yaogang1985@163.com
}

Yang Libin

Department of Ordnance Science and Technology

Naval Aeronautical and Astronautical University

Yantai, China

e-mail: hyylb201@163.com

\begin{abstract}
Traditional error separation methods could not tackle systematic error with complicated transformation law in measuring system very well, so that we present an improved Empirical Mode Decomposition (EMD). The end effect existed in the course of EMD decomposition, endpoints continuation based on the degree of waveform similarity was applied to restrain the end effect. As to EMD decomposition termination criterions, it was difficult for EMD method to determine appropriate termination criterions, cumulative percent variance was introduced to determine two decomposition termination criterions. Given superposition of multiple types of systematic errors, the result of simulation example shows that the proposed method exceeds others, as its error is the least. Finally, the example including dynamic goniometer error data implies that EMD brings a good effect on the separation.
\end{abstract}

Keywords-Empirical Mode Decomposition; systematic error separation; degree of waveform similarity; end effect; cumulative percent variance

\section{INTRODUCTION}

There are errors existing in all the measuring system. The value of error, measured value minus true value, can have a direct impact on the accuracy of the measurement system. Measurement error contains random and systematic errors. The characteristic of random errors is uncertain, and its law cannot be determined, but can be studied by statistical methods. However, systematic errors can be predicted by its laws of change. In order to improve the accuracy of measuring system, the further analysis of systematic errors needs to be made, and the key point is the separation of systematic error. For complex systems, the systematic error model is difficult to identify, the influence of result is difficult to estimate too.

Systematic error contains constant error, linear drift error, periodic error and complex changes errors. The separation of systematic error can be considered as the processes that different frequency band of error signal are separated. Currently, Separation methods of measurement errors mainly include east-squares regression method, median filtering, Fourier series approximation method, wavelet and wavelet packet decomposition method and so on $^{[1]}$. But there are some limitations in these methods, such as the moment of model is difficult to determined in the classical least squares regression method; the accuracy of median filtering is difficult to improve; Fourier series approximation is harmonic decomposition of error; the quality of the separation of wavelet decomposition and wavelet packet is determined by wavelet function. Above all, the improved EMD method is researched to analyze the systematic error, and demonstrated through examples.

\section{FUNDAMENTALS OF EMD}

\section{A. Overview}

In 1998, time-frequency analysis method based on EMD (Empirical Mode Decomposition) was presented by N. E. Huang, and it was a new signal processing method $^{[2][3]}$. It can deal with non-linear and non-stationary signal, base function is uncertainty in the decomposition, and depends only on the data itself, which has remarkable self-adaptability. It is considered that data consists of one or more IMF (Intrinsic Mode Functions) The method has been applied in the field of biology, ocean, earth science, astronomy and engineering technology because of its advantages. Systematic errors are non-linear and non-stationary in the real world and the EMD can provide a new way to deal with complex and volatile errors.

The characters of each IMF decomposed by EMD method are as follows:

(1) in the entire signal, number of extreme points and zero crossing point are equal or a differential of one at most; 
(2)Local mean value is zero, which is the mean value of envelope consists of local maximum envelope and local minimum envelope equals to zero.

In practice, the mean value of upper and lower envelopes cannot be zero, so if the following inequality is true, the mean value of envelope is considered to satisfy the condition the mean value of IMF is zero:

$$
\frac{\sum\left[h_{1 k-1}(t)-h_{1 k}\right]^{2}}{\sum\left[h_{1 k-1}(t)\right]^{2}} \leq \varepsilon
$$

where $\varepsilon$ is screening threshold, its value is between 0.2 and 0.3 , the mean of other notions can be found in section 2.2 .

\section{B. EMD Decomposition Process}

EMD algorithm assumes that any signal is consisted of a certain number of intrinsic mode functions, each intrinsic mode function can be obtained by following $\operatorname{methods}^{[3][5]}$, it is supposed that the original signal is $x(t)$ :

(1) The maxima of the signal $x(t)$ would be found, the upper and lower envelope of the signal can be fitted by cubic spline, which are $e \max _{k}$ and $e \min _{k}$ respectively.

(2) The mean value of the upper and lower envelope is calculated as follows:

$$
e m_{k}=\left(e \max _{k}+e \min _{k}\right) / 2
$$

(3) A new signal can be obtained by the different between original signal and the mean of envelope:

$$
h_{k 1}=x-e m_{k}
$$

Whether $h_{k 1}$ can meet the IMF's conditions is needed to determine: if true, $h_{k 1}$ is an IMF component, and can be denoted by $i m f_{1}$, so $h_{k 1}=x-i m f_{1}$ would be decomposed any further; if false, $h_{k i}$ would be decomposed any further as original signal until the first IMF component $i m f_{1}$ is found.

$\operatorname{imf}_{2}, \ldots, i m f_{n}$ can be obtained by Repeating above steps, the decomposition would be stopped until remainder term $r_{n}$ is obtained. And $r_{n}$ is a monotonic signal or less than a given threshold in advance.

The original function can be expressed by EMD decomposition as follows:

$$
x(t)=\sum_{i=1}^{n} i m f_{i}+r_{n}
$$

\section{Endpoints Continuation Based on the Waveform Similarity}

\section{1) Principle of endpoint effect}

When the error signal is decomposed with EMD, the end effect is key problem to be solved. The reason of the end effect generation is that extreme points need to fit when the upper and lower envelopes of signals are calculated. If endpoints of signals are considered directly as extreme points of signals, the fitted envelopes can cover the whole signals. Although the conditions of empirical mode decomposition can be met, the big error will be caused near the envelopes of endpoints, and with the constantly repeated screening process, the whole decomposition will be influenced because of error propagation to signal. For longer data, the end effect can be eliminated in the way of discarding two endpoints data in decomposition. But for short data, it can only be eliminated with continuation.

Currently, there are some suppression methods of the end effect including the mirror continuation method, continuation method based on neural network, continuation method based on polynomial fitting [6] [7] ${ }^{[8][9]}$.However, the methods have some disadvantages. For the mirror continuation method, its effect in dealing with short data is poor, because it may have to truncate some data. For continuation method based on neural network, its drawback is that the speed is too slow. For continuation method based on polynomial fitting, it is poor adaptability. Thus, the continuation method based on waveform similarity is proposed.

2) Endpoints continuation based on waveform similarity

The suppression principle of end effect is to look for the extreme point closest to the endpoint signal, which outside the signal. And it together extreme points of signals can be the upper and lower envelopes with spline interpolation, then the decomposition data will be intercepted. Continuation is a method to deal with all kinds of data, and predict the trend of signal in the endpoint. If some waveforms in signal are similar to the waveforms in the endpoint, the data relationship can be set up to extend the original signal based on the similarity of the data.

\section{3) Waveform similarity}

Waveform similarity is a measure to show the similarity of two waveforms. For signal $x(t)$ and $y(t)$, $t=1,2 \ldots n$, the similarity of two waveforms can be defined as

$$
\rho(x(t), y(t))=\frac{\operatorname{cov}(x(t), y(t))}{\sqrt{\sigma(x) \sigma(y)}}
$$

Where $\operatorname{cov}(\cdot)$ is covariance of $\mathrm{x}(\mathrm{t})$ and $\mathrm{y}(\mathrm{t}), \sigma(x)$ and $\sigma(y)$ are variance of $\mathrm{x}(\mathrm{t})$ and $\mathrm{y}(\mathrm{t})$ respectively.

The range of $\rho$ is $[-1,1],|\rho|$ represents the similarity of them, and the bigger $|\rho|$ it is, the more similarity they are. When $\rho$ equals 1 , it most similarity the waveforms are, and the same peak direction; When $\rho$ equals -1 , the waveforms are similar but inversion, and the peak direction are opposite

Theorem 1: If and only if there is a linear relationship $Y=a+b X$ between the random variables $\mathrm{Y}$ and $\mathrm{X}$, the absolute value of the correlation coefficient $\rho(X, Y)$ is equal to 1 . 
According to Theorem 1, when the similarity of two waveforms is high ( $\rho \geq 0.8$ ), it is approximately considered that the relationship of them is linear. Their linear relationship can be determined in linear regression method, where the unknown parameters $a$ and $b$ can be estimated in the least squares method

\section{4) Steps of continuation}

Take the left endpoint for example to show continuation as the left and right endpoint is the same:

Step 1: Determining the matching wave and its length. It is assumed that the matching wave is $\omega$, and its starting point is the value of left endpoint, to the right, it includes a maximum and minimum values. When the distance from the first maxima to the left endpoint $L_{\max 1}$ is less than that from the first minimum value to the left point $L_{\min 1}$, and vice versa.

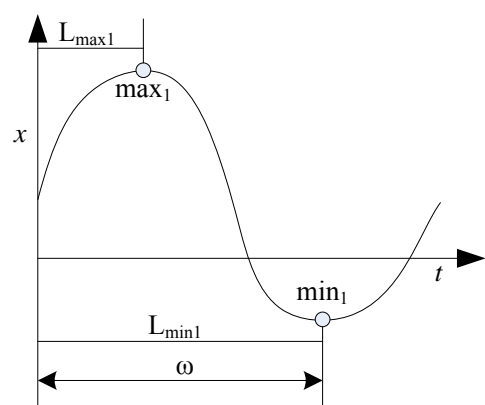

Figure 1. An example of the matching wave

Step 2: Calculating the similarity $\rho$ of $\omega$ and waveforms in the whole data segment. In order to increase computing speed, the similarities of the waves nearby the extreme points are calculated only.

Step 3: Determining the highest similarity sub band, i.e. the largest waves of $|\rho|$.

Step 4: Calculating data relationship of the two bands in linear regression method:

$$
Y=a+b X
$$

Where $X$ is amplitude vector of $\omega, Y$ is amplitude vector of $\omega$ match

Step 5: Finding the maximum value $\left(t_{\max 0}, \max _{0}\right)$ and the minimum value $\left(t_{\min 1}, \min _{1}\right)$ respectively with left continuation. It is assumed that the maximum value and the minimum value nearby the left of $\omega_{-}$match are $\left(t_{\max p}, \max _{p}\right)$ and $\left(t_{\min p}, \min _{p}\right)$ respectively. The amplitude of the maximum value $\max _{0}$ and the minimum value $\min _{0}$ can be determined as follows:

$$
\begin{aligned}
\max _{0} & =\left(\max _{p}-b\right) / a \\
\min _{0} & =\left(\min _{p}-b\right) / a
\end{aligned}
$$

The time corresponding to continuation is determined by distance from extreme value to endpoint. It is assumed that the distance from $\left(t_{\max p}, \max _{p}\right)$ and $\left(t_{\min p}, \min _{p}\right)$ to $\omega_{-}$match are $L_{\max p}$ and $L_{\min p}$, so the time corresponding to the maximum value and the minimum value are respectively:

$$
\begin{aligned}
& t_{\text {max } 0}=-L_{\max P} \\
& t_{\min 0}=-L_{\min P}
\end{aligned}
$$

\section{EMD Decomposition TerminationCriterion}

EMD decomposition termination condition is the criterion to stop EMD. When the termination conditions are too harsh, excessive IMF component will be produced, which can interfere the correct judgment of the signal; when the termination conditions are loose, the decomposition is not meticulous, and the law of signal will not be identified. For the whole decomposition process of EMD, the judgment to how to terminate decomposition need to be made. The common two criteria are: first, when the amplitude of intrinsic mode function or the residual component is less than the default value, the entire decomposition process stops; second, when the residual component becomes a monotonic function or constant, and the intrinsic mode functions cannot be screened out, the entire decomposition process stops. Proposed principal component analysis (PCA) to determine the decomposition termination time by cumulative variance contribution rate, and IMF and the residual component are need to make principal component analysis. PCA is to project the original variables into a new coordinate, so that the new PCAs are unrelated, and the original variables can be represented by few dimensions. The IMFs obtained by EMD method are approximately orthogonal, but not approximately correlative, so the cumulative variance contribution rate can be calculated directly.

\section{1) Approximate orthogonality of IMF}

From the EMD decomposition process, the orthogonality in a practical sense is satisfied. But until now, a rigorous mathematical proof has not been given in theory. Each intrinsic mode function obtained by the EMD should be mutually orthogonal on the local, because each intrinsic mode functions are obtained by the difference of the original signal and the local mean of its maximum and minimum values, so

$$
\overline{(y(t)-\overline{y(t)}) \overline{y(t)}}=0
$$

Where $\overline{y(t)}$ is mean of $y(t)$.

In the strict sense, the above formula is not accurate, because the mean value is calculated by curve fitting maxima and minima of signal, so there is some error with the true mean value, but still small. The orthogonality of IMF can be given by way of posteriori. The original signal can be reconstructed as:

$$
x(t)=\sum_{i=1}^{n+1} i m f_{i}
$$


Where the residual component $r_{n}(t)$ can be seen as the $n+1_{\text {th }}$ IMF components, and then

$$
x^{2}(t)=\sum_{i=1}^{n+1} i m f_{i}^{2}+2 \sum_{i=1}^{n+1} \sum_{j=1}^{n+1} i m f_{i}(t) i m f_{j}(t)
$$

Therefore, the orthogonality index of the original signal $x(t)$ (Index of Orthogonality) can be defined as:

$$
I O=\sum_{t=0}^{T}\left(\sum_{i=1}^{n+1} \sum_{j=1}^{n+1} i m f_{i}(t) i m f_{j}(t) / x^{2}(t)\right)
$$

where $T$ is the length of signal.

If the decomposition is orthogonal, then the cross term of equation(14) is zero. Through a lot of tests, a conclusion validated by Huang et al is that orthogonality indicators of the common signal are usually no more than $1 \%$, orthogonality indicators may reach $5 \%$ in extreme cases for transient signals, in other words, each intrinsic mode function decomposed by EMD is nearly orthogonal.

2) Decomposition Termination Criterion Based on Cumulative Variance Contribution Rate

The concept of variance contribution rate derives from PCA. Variance contribution rate and the cumulative variance contribution rate are key indicators of the main ingredients. In the improved EMD method, some defines are given as follows:

The variance contribution rate of $i_{\text {th }}$ IMF is:

$$
\text { Contr }_{i}=\frac{\sigma\left(\text { imf }_{i}\right)}{\sum_{j=1}^{m} \sigma\left(i m f_{i}\right)} \times 100 \%
$$

The bigger value is, the more information $i_{\text {th }}$ IMF covers. And $\operatorname{var}\left(i m f_{i}\right)$ is the variance of $i_{\text {th }} \mathrm{IMF}$.

Cumulative Percent Variance is the sum of the former $\mathrm{r}$ IMF variance contribution rates. In order to reflect fluctuation variables better, in general, the threshold greater than an expectation should be chosen.

$$
C P V(r)=\sum_{i=1}^{r} \operatorname{Contr}(i)=\frac{\sum_{i=1}^{r} \sigma\left(\operatorname{imf}_{i}\right)}{\sum_{j=1}^{n+1} \sigma\left(\operatorname{imf}_{i}\right)} \times 100 \% \geq C L
$$

where $C L$ is the determined threshold value.

According to the approximate orthogonality of IMF components, the information content of each IMF components can be measured in variance contribution rate. According to the decomposition termination time of the cumulative variance contribution rate, the variance contribution rate of each decomposed IMF components $i m f_{1}, i m f_{2}, \Lambda i m f_{n}$ and the residual component $r_{n}$ can be calculated. If the contribution rate of the residual component is less than the given threshold, the decomposition is terminated. If the variance contribution rate of each components is less than $4 \times 10^{-3}$, then
$C L \geq 1-4 \times 10^{-3}$. According to the above criterion, two decomposition termination criterions are applied: first, when the cumulative variance contribution rate is greater than the given threshold, the entire decomposition process stops; second, when the residual component becomes a monotonic function or a constant, the intrinsic mode functions cannot be screened out, the entire decomposition process stops.

\section{SiMULATION EXAMPLES}

\section{A. Superposition of Multiple Types of Systematic Error}

Manifestations of systematic errors can be a single constant error, linear drift error or periodic error, and can also be a combination between them. In this paper, two forms of error superimposition are researched to illustrate the effectiveness of EMD method. It is Assumed that systematic error contains three error components, which $y_{1}(t)$ is a linear error (constant error can be regarded as a special form of linear errors), $y_{2}(t), y_{3}(t)$ are two periodic errors. Each error can be expressed as follows:

$$
\begin{gathered}
\qquad y(t)=y_{1}(t)+y_{2}(t)+y_{3}(t) \\
\text { where }\left\{\begin{array}{l}
y_{1}(t)=a x+b, a=0.5, b=1 \\
y_{2}(t)=2 \sin (2 \pi t) \\
y_{3}(t)=\sin (10 \pi t)
\end{array} \quad t=0: 0.001: 3\right.
\end{gathered}
$$

In order to compare the effectiveness of waveform similarity endpoint continuation methods, the EMD decomposition, the mirror continuation decomposition method and the proposed method are researched on the above signal respectively, the results are as follows.
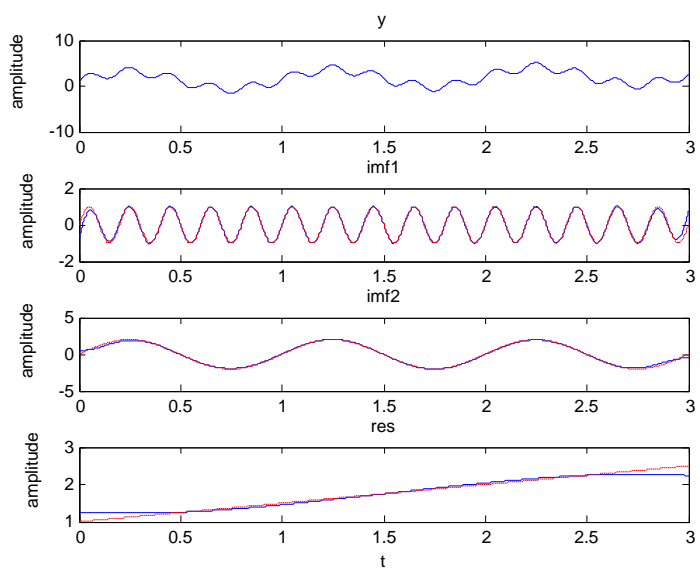

Figure 2. Decomposition by EMD 

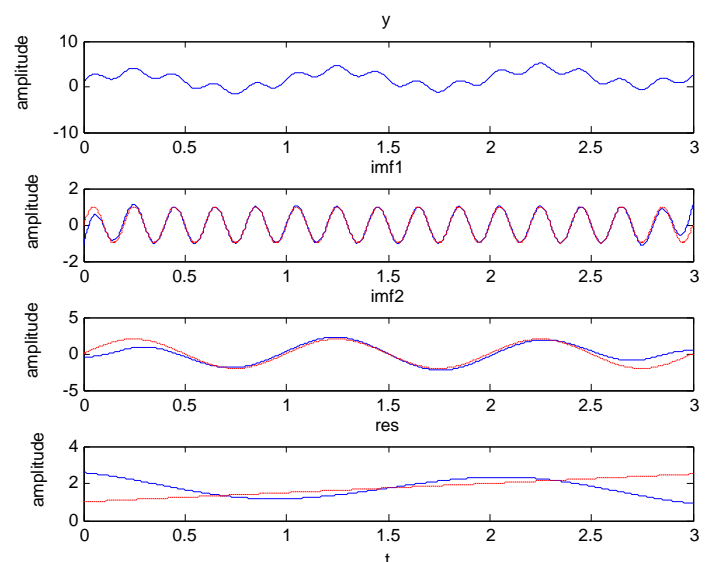

Figure 3. Decomposition by Mirror continuation method
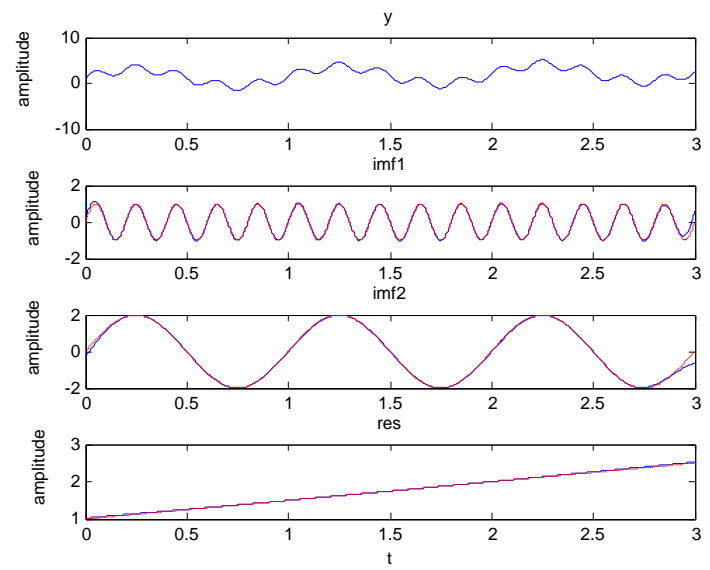

Figure 4. Decomposition by endpoints continuation based on the waveform similarity

TABLE1. MSE OF ALL THE THREE DECOMPOSITION METHODS

\begin{tabular}{|l|l|l|l|}
\hline $\begin{array}{l}\text { signal } \\
\text { methods }\end{array}$ & $y_{1}(t)$ & $y_{2}(t)$ & $y_{3}(t)$ \\
\hline $\begin{array}{l}\text { EMD } \\
\text { decomposition }\end{array}$ & 0.0818 & 0.0979 & 0.0975 \\
\hline $\begin{array}{l}\text { Mirror } \\
\text { continuation } \\
\text { decomposition }\end{array}$ & 0.6597 & 0.6221 & 0.1656 \\
\hline $\begin{array}{l}\text { The proposed } \\
\text { method }\end{array}$ & 0.0096 & 0.0730 & 0.0693 \\
\hline
\end{tabular}

According to the above figure, two IMF components and a residual component can be obtained by three methods. The error of the original signal and the signal decomposed by EMD is small; the biggest error of three components is a linear error, because of the end effects spreading to the inside; for Mirror continuation decomposition, as a result of irrational the mirror point selection, a false component is produced, which lead to a big error. The three components are decomposed by waveform similarity continuation EMD decomposition method, the decomposition effect in the endpoint is good, and the error is the least.

\section{B. Coexistence of Random and Systematic Errors}

In a real measurement system, the error contains random and systematic errors. Conducting random errors is the first step of separating systematic errors. The above signal plus normal white noise in simulation, and the experimental results can be shown as follows.

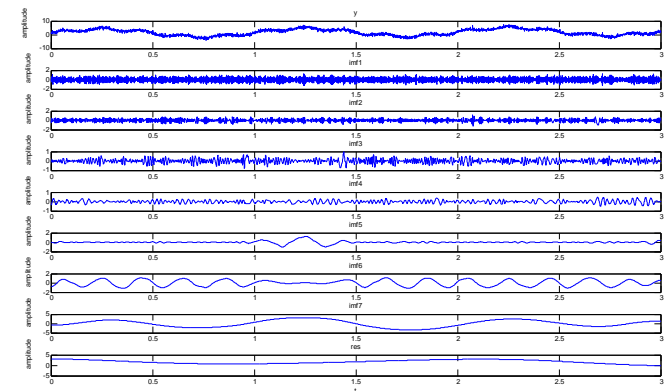

Figure 5. Directe composition by EMD
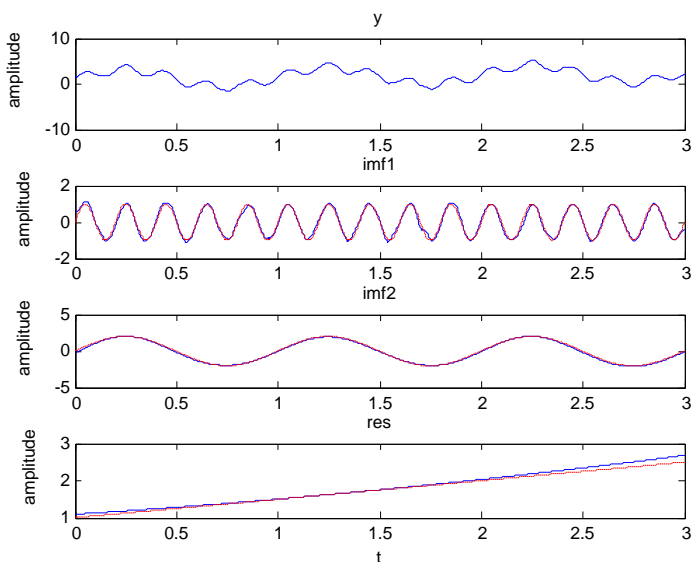

Figure6. Decomposition by endpoints continuation based on the waveform similarity after the denoising

Fig . 4 is a result of not processing the random error in decomposition, a lot of high-frequency IMF components are produced due to the higher frequency of random errors in decomposition, so that the decomposition process is disturbed, and systematic errors are not separated very well. As Fig .5 shown, it is not only systematic errors can be separated completely, but also the end effect has been well suppressed.

\section{EXAMPLE ANALYSIS}

The separation effect of the improved EMD method can be verified through the dynamic goniometer error data in Schedule 5 of [1]. Given the small number of sampling points, error curve obtained by spline interpolation is shown as Fig .6. The result decomposed by EMD is shown as Fig .7. According to Fig .7, the original system errors contain linear drift error and periodic errors. Four errors can be separated by improved EMD method, where imf1, imf2, imf3 are cyclical error harmonics, and the residual component is a linear drift error. 


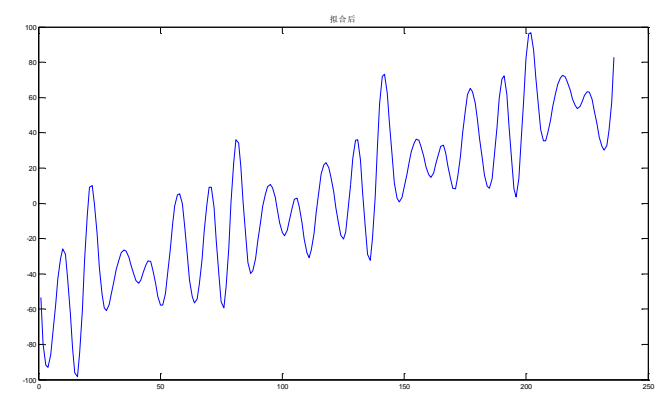

Figure 7.Error of dynamic goniometer
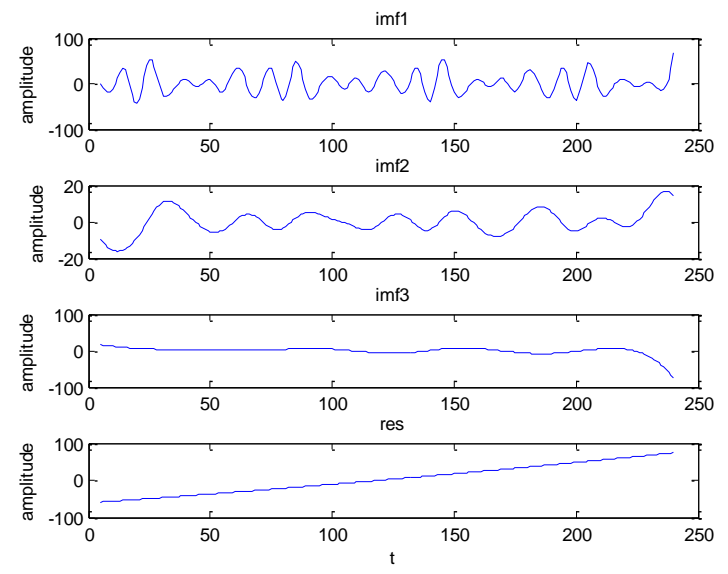

Figure 8. Decomposition by the improved EMD method

\section{CONCLUSION}

EMD method is a new data processing method. It can not only handle linear, smooth data, but also can handle nonlinear and non-stationary data adaptively. In this paper, the improved EMD method is applied to the systematic errors separation process. As simulation experiments and example analysis shown, the end effect of EMD can be restrained by endpoint continuation method based on waveform similarity, the prospered composition time can be determined by variance contribution rate, and systematic errors separation can be done well, so it has a good prospect of application.

\section{REFERENCES}

[1] Li Shipin,Fu Yu,Zhang Jing, "Systematic error separation method based on EMD," China Measurement\&Test, vol. 37, 2011, pp.9-13.

[2] N. E. Huang, Z. Shen, S. R. Long, et al, "Theempiricalmode decomposition method and the Hilbert spectrum for non-stationary time series analysis,” Proc. R. Soc. London, U.K.:[s.n.], 1998, pp. 903-995.

[3] Norden E. Huang, "Review of Empirical Mode Decomposition," Proceedings of SPIE. Olando, USA:[s.n.], 2001, pp. 71-80

[4] Gabriel Rilling,Patrick Flandrin,Paulo Goncalves, "On Empirical Mode Decomposition and its Algorithms," IEEE-EURASIP Workshop on Nonlinear Signal and Image Processing, Grado, Italy:[s.n.], 2003, pp. 811-815.

[5] Jian Zhang, Ruqiang Yan, RobertX.Gao, et al, "Performance enhancement of ensemble empirical mode decomposition," Mechanical Systems and Signal Processing, 2010, pp. 2104-2123.

[6] Wang Ting, "Research on EMD algorithm and its application in signal denoising," Harbin:Harbin Engineering University, 2010.

[7] Shao Chenxi,Wang Jian,Fan Jinfen,et al, "A self-adaptive method dealing with the end issue of EMD," Acta Electronnica Sinica, vol. 35, 2007, pp. 1944-1948.

[8] Guo Mingwei, Ni Shihong, Zhu Jiahai, et al, "HHT/EMD end extension method in vibration signal analysis," Journal of vibration and Shock, vol. 31, 2012, pp. 62-66.

[9] Xu Baojie, Zhang Jianmin, Xu Xiaoli, et al, "A Study on the method of restraining the ending effect of empirical mode decomposition," Transactions of BeijingInstitue of Technolog, vol. 26, 2006, pp. 196-200.

[10] Fei Yetai, Lu Shenrong, "The priciple and technique of dynamic measurement error correction," Beijing:China metrology Publishing Company, 2001. 